\title{
ON DUALITY FOR CONVEX MINIMIZATION WITH NESTED MAXIMA
}

\author{
C. H. SCOTT ${ }^{1}$, T. R. JEFFERSON ${ }^{2}$ AND E. SIRRI ${ }^{1}$
}

(Received 15 September 1983; revised 29 February 1984)

\begin{abstract}
In this paper, we consider convex programs with linear constraints where the objective function involves nested maxima of linear functions as well as a convex function. A dual program is constructed which has interpretational significance and may be easier to solve than the primal formulation. A numerical example is given to illustrate the method.
\end{abstract}

\section{Introduction}

Consider the following nondifferentiable convex program.

$$
\begin{gathered}
\text { (P): } \underset{x \in R^{n}}{\operatorname{Minimize}} f(x)+\underset{i \in I}{\operatorname{Max}}\left(a_{i}^{T} x+b_{i}\right) \\
\text { subject to } C x \leqslant d .
\end{gathered}
$$

Here $f(\cdot)$ is a closed convex function defined over $R^{n}, I$ is a finite dimensional index set and $a_{i}, i \in I ; b_{i}, i \in I ; C$ and $d$ are given parameters. Such programs arise naturally in a variety of settings where one is trading off convex exploitation and linear investment costs. It is the purpose of this paper to construct a dual and duality relations for the above program and to examine its significance. The mathematical machinery is provided by the generalized theory of geometric programming [3] which is summarized in Section 2 below. In Section 3, this duality theory is used to develop a dual for program (P) and its significance discussed. A numerical example, solved by MINOS [2] is given in Section 4.

\footnotetext{
${ }^{1}$ Graduate School of Management, University of California, Irvine, California 92715, U.S.A.

${ }^{2}$ Department of Industrial Engineering, University of Pittsburgh, Pittsburgh, Pennsylvania 15261, U.S.A.

Copyright Australian Mathematical Society 1985, Serial-fee code 0334-2700/85
} 


\section{Generalized geometric programming}

We consider an optimization problem of the form:

$$
\begin{gathered}
\text { Minimize } f(x), \quad x \in D \\
\text { subject to } x \in \chi .
\end{gathered}
$$

where $\chi$ is a closed convex cone in $R^{n}$ and $f$ is a closed convex function defined on a closed convex set $D$ in $R^{n}$. This is termed the primal problem. We associate with the primal problem, another problem called the dual problem, which is of the form:

$$
\begin{gathered}
\text { Minimize } f^{*}\left(x^{*}\right), \quad x^{*} \in D^{*} \\
\text { subject to } x^{*} \in \chi^{*},
\end{gathered}
$$

where $\chi^{*}$ denotes the dual cone of $\chi$ in $R^{n}$ and $\left[f^{*}: D^{*}\right]$ is the conjugate transform of $[f: D]$ defined by

$$
f^{*}\left(x^{*}\right)=\sup _{x \in D}\left(x^{T} x^{*}-f(x)\right)
$$

and

$$
D^{*}=\left\{x^{*} \mid \sup _{x \in D}\left(x^{T} x^{*}-f(x)\right)<+\infty\right\}
$$

$T$ denotes a transpose and

$$
x^{*}=\left\{x^{*} \mid x^{T} x^{*} \geqslant 0, \forall x \in \chi\right\} .
$$

At optimality, Peterson [3] has shown that the following relationships hold between the primal and dual optimal points $x_{0}$ and $x_{0}^{*}$ respectively.

$$
\begin{gathered}
f\left(x_{0}\right)+f^{*}\left(x_{0}^{*}\right)=0, \\
x_{0}^{*} \in \partial f\left(x_{0}\right), \quad x_{0} \in \partial f^{*}\left(x_{0}^{*}\right), \\
x_{0}^{T} x_{0}^{*}=0 .
\end{gathered}
$$

Here $\partial f\left(x_{0}\right)$ denotes the subgradient set of $f$ at $x_{0}$ and is defined by

$$
\partial f\left(x_{0}\right)=\left\{x^{*} \mid f(z) \geqslant f\left(x_{0}\right)+x_{0}^{* T}\left(z-x_{0}\right), \forall z \in D\right\} .
$$

In the case where $f(x)$ is a differentiable function, the subgradient set is replaced by the gradient, that is, (11) may be written

$$
x_{0}^{*}=\nabla f\left(x_{0}\right), \quad x_{0}=\nabla f^{*}\left(x_{0}^{*}\right),
$$

where $\nabla$ denotes the gradient operator.

These optimality conditions allow an optimal point for one program to be calculated from an optimal point of the other. 


\section{The dual program}

Program (P) defined by (1) and (2) may be written in the following equivalent form which is more amenable to analysis.

$$
\begin{gathered}
\left(\mathrm{P}^{\prime}\right): \underset{x, \alpha}{\operatorname{Minimize}} f(x)+\alpha \\
\text { subject to } a_{i}^{T} x+b_{i} \leqslant \alpha, \quad i \in I \\
C x \leqslant d .
\end{gathered}
$$

With the introduction of a scalar variable $\alpha$, the program is no longer nondifferentiable, however, additional linear constraints have been added. In order to invoke generalized geometric programming duality, we need to transform the constraints (16) and (17) into a cone. To this end, we introduce new variables $\beta$ and $\gamma$ restricted to one point domains $b$ and $d$, respectively. Hence program $\left(\mathrm{P}^{\prime}\right)$ may be equivalently written as

$$
\begin{gathered}
\left(\mathrm{P}^{\prime \prime}\right): \underset{x, \alpha, \beta \in\{b\}, \gamma \in\{d\}}{\operatorname{Minimize}} f(x)+\alpha \\
\text { subject to } \alpha-a_{i}^{T} x-\beta_{i} \geqslant 0, \quad i \in I \\
\gamma-C x \geqslant 0 .
\end{gathered}
$$

The dual objective is given by the conjugate transform of (18). This is

$$
\begin{aligned}
\sup _{x, \alpha, \beta \in\{b\}, \gamma \in\{d\}}\left(x^{T} x^{*}+\alpha^{T} \alpha^{*}+\beta^{T} \beta^{*}+\gamma^{T} \gamma^{*}-f(x)-\alpha\right) \\
=f^{*}\left(x^{*}\right)+b^{T} \beta^{*}+d^{T} \gamma^{*} \quad \text { with } \alpha^{*}=1 \text { and } x^{*} \in S .
\end{aligned}
$$

Here $S$ is the domain of $f^{*}(\cdot)$ which will usually be $R^{n}, R_{+}^{n}$ or $R_{-}^{n}$.

Further we require the dual of the cone generated by (19) and (20). This is given by

$$
\left(\begin{array}{c}
x^{*} \\
\alpha^{*} \\
\beta^{*} \\
\gamma^{*}
\end{array}\right)=\left(\begin{array}{r}
-A^{T} \\
U \\
-I \\
0
\end{array}\right) u+\left(\begin{array}{c}
-C^{T} \\
0 \\
0 \\
I
\end{array}\right) v
$$

where $A=\left\|a_{i j}\right\|, U=(1,1, \ldots, 1)$ and $u \geqslant 0, V \geqslant 0$. It follows that

$$
\begin{gathered}
x^{*}=-A^{T} u-C^{T} v, \\
\alpha^{*}=\sum_{i \in I} u_{i}, \\
\beta^{*}=-u \geqslant 0, \\
\gamma^{*}=v \geqslant 0 .
\end{gathered}
$$


Collecting results (21)-(26), the following dual results

$$
\text { (D): } \underset{u, v}{\text { Minimize }} f^{*}\left(-A^{T} u-C^{T} v\right)-b^{T} u+d^{T} v
$$

subject to

$$
\begin{gathered}
\sum_{i} u_{i}=1, \\
-A^{T} u-C^{T} v \in S, \\
u \geqslant 0, \quad v \geqslant 0 .
\end{gathered}
$$

Further, at optimality, the primal and dual variables are related by

$$
\begin{aligned}
f(x)+\operatorname{Max}_{i \in I}\left(a_{i}^{T}+b_{i}\right)+f^{*}\left(-A^{T} u-C^{T} v\right)-b v^{T}+d u^{T}=0, \\
-A u^{T}-C v^{T} \in \partial f(x), x \in \partial f^{*}\left(-A^{T} u-C^{T} v\right), \\
u_{i}\left(a_{i}^{T} x+b_{i}-\alpha\right)=0, \quad i \in I, \\
v_{k}\left(\sum C_{k j} x_{j}-d_{k}\right)=0, \quad \forall k .
\end{aligned}
$$

Here the dual variable $v$ has the usual interpretation of the shadow price associated with the resource availability of the righthand side of (2). Similarly the dual variable $u_{i}$ has the interpretation of the shadow price associated with $b_{i}$. However, specifically it indicates, when strictly positive, those terms in the nested maxima which contributes to the optimal objective value. This follows as a consequence of complementary slackness.

The computational viability of the dual program (D) depends on the availability in analytic form of the function $f^{*}(\cdot)$ and on the structure of the set $S$. The former question has been discussed elsewhere (e.g. [1], [4]); in the latter case $S$ is often the whole space of an orthant. For example, when $f(x)=\sum_{i=1}^{n} c_{i} \exp x_{i}$, it may be shown that $S=R_{+}^{n}$. In this case of the quadratic function $f(x)=\sum c_{i} x_{i}^{2}$, the dual is particularly simple since $S$ is the whole space and program (D) has essentially one constraint, namely equality (28). A numerical example, where the dual is solved by MINOS, is given in the final section.

\section{A numerical example}

Consider the program

$$
\begin{aligned}
& \underset{x}{\operatorname{Minimize}}\left[8 x_{1}^{2}+5 x_{2}^{2}+7 x_{3}^{2}+8 x_{4}^{2}\right. \\
& +\operatorname{Max}\left(2 x_{1}+3 x_{2}+5 x_{3}+x_{4},\right. \\
& \left.\left.x_{1}+2 x_{2}+x_{3}+2 x_{4}, 3 x_{1}+x_{3}+3 x_{4}\right)\right]
\end{aligned}
$$


subject to

$$
\begin{aligned}
& x_{1}+x_{4} \geqslant 10 \\
& x_{1}+2 x_{2}+x_{3} \geqslant 5 \\
& -2 x_{2}+5 x_{4} \geqslant 31 \\
& 5 x_{3} \geqslant 28 \\
& 12 x_{1}+2 x_{3}-x_{4} \geqslant 17 \text {. }
\end{aligned}
$$

A straightforward calculation shows the dual program to be

$$
\begin{aligned}
\underset{u}{\operatorname{Minimize}}[1 / 32 & \left.2 u_{1}+u_{2}+3 u_{3}+v_{1}+v_{2}+12 v_{5}\right)^{2} \\
+ & 1 / 20\left(3 u_{1}+2 u_{2}+u_{3}+2 v_{2}-2 v_{3}\right)^{2} \\
+ & 1 / 28\left(5 u_{1}+u_{2}-u_{3}+v_{2}+5 v_{4}+2 v_{5}\right)^{2} \\
+1 / 32\left(u_{1}+\right. & \left.2 u_{2}-u_{3}+v_{1}+5 v_{3}-v_{5}\right)^{2} \\
& \left.+10 v_{1}+5 v_{2}+31 v_{3}+28 v_{4}+17 v_{5}\right]
\end{aligned}
$$

subject to

$$
\begin{gathered}
u_{1}+u_{2}+u_{3}=1 \\
u \geqslant 0, \quad v \geqslant 0 .
\end{gathered}
$$

With only one linear equality constraint and nonnegativity conditions, this is a particularly simple nonlinear program. The solution, from MINOS, is found to be

$$
\begin{gathered}
u_{1}=1, \quad u_{2}=0, \quad u_{3}=0, \\
v_{1}=70.40212, \quad v_{2}=0, \quad v_{3}=4.439153, \quad v_{4}=16.68, \quad v_{5}=0,
\end{gathered}
$$

with objective value -673.69328042 .

From (44), it follows that the only linear term contributing to the minimal objective value is $i=1$. Using the relations between the primal and dual variables in (31) to (34), it follows that the optimal primal solution is

$$
x_{1}=4.275132, \quad x_{2}=1.187831, \quad x_{3}=5.6, \quad x_{4}=5.724868
$$

with objective value 673.69328042 .

\section{Acknowledgements}

The authors are grateful to a referee who pointed out that the same results may be derived via Lagrangian duality.

This research was sponsored by the United States Air Force Office of Scientific Research under AFOSR grant number 83-0234. 


\section{References}

[1] W. Fenchel, Convex cones, sets and functions (Princeton University, 1953).

[2] B. A. Murtagh and M. A. Saunders, "Large scale linearly constrained optimization", Math. Programming 14 (1978), 44-72.

[3] E. L. Peterson, "Geometric programming", SIAM Rev. 18 (1976), 1-52.

[4] R. T. Rockafellar, Convex analysis (Princeton University, 1970). 